\title{
Preoperative muscle weakness as defined by handgrip strength and postoperative outcomes: a systematic review
}

Pervez Sultan ${ }^{1}$, Mark A Hamilton ${ }^{2}$ and Gareth L Ackland ${ }^{3,4^{*}}$

\begin{abstract}
Background: Reduced muscle strength- commonly characterized by decreased handgrip strength compared to population norms- is associated with numerous untoward outcomes. Preoperative handgrip strength is a potentially attractive real-time, non-invasive, cheap and easy-to-perform "bedside" assessment tool. Using systematic review procedure, we investigated whether preoperative handgrip strength was associated with postoperative outcomes in adults undergoing surgery.

Methods: PRISMA and MOOSE consensus guidelines for reporting systematic reviews were followed. MEDLINE, EMBASE, and the Cochrane Central Register of Controlled Clinical Trials (1980-2010) were systematically searched by two independent reviewers. The selection criteria were limited to include studies of preoperative handgrip strength in human adults undergoing non-emergency, cardiac and non-cardiac surgery. Study procedural quality was analysed using the Newcastle-Ottawa Quality Assessment score. The outcomes assessed were postoperative morbidity, mortality and hospital stay.

Results: Nineteen clinical studies (17 prospective; 4 in urgent surgery) comprising 2194 patients were identified between 1980-2010. Impaired handgrip strength and postoperative morbidity were defined inconsistently between studies. Only 2 studies explicitly ensured investigators collecting postoperative outcomes data were blinded to preoperative handgrip strength test results. The heterogeneity of study design used and the diversity of surgical procedures precluded formal meta-analysis. Despite the moderate quality of these observational studies, lower handgrip strength was associated with increased morbidity ( $n=10$ studies), mortality ( $n=2 / 5$ studies) and length of hospital stay ( $n=3 / 7$ studies).

Conclusions: Impaired preoperative handgrip strength may be associated with poorer postoperative outcomes, but further work exploring its predictive power is warranted using prospectively acquired, objectively defined measures of postoperative morbidity.
\end{abstract}

\section{Background}

A substantial minority of patients sustain an excess of postoperative complications [1] and accelerated, posthospital discharge mortality [2]. In surgical procedures known to have a mortality of greater than $5 \%$ in the UK, elderly patients (mean age 75 years) and emergency procedures account for over $80 \%$ of deaths but less than $15 \%$ of total procedures [3]. Physician- and patient-friendly, practical and inexpensive tools are required to guide and

\footnotetext{
* Correspondence: g.ackland@ucl.ac.uk

${ }^{3}$ Centre for Anaesthesia, Critical Care and Pain Management, University

College London, London, UK

Full list of author information is available at the end of the article
}

risk-stratify perioperative management objectively for this cohort of patients. Measurements of exercise capacity and muscle strength are associated with increased all-cause and cardiovascular mortality in the general population [4-7]. However, the comprehensive assessment of cardiovascular reserve - most objectively using cardiopulmonary exercise testing [8] - is challenging for immobile patients, time-consuming, and costly to extend as a general screening tool to the wider, at-risk surgical population. By contrast handgrip strength is an inexpensive, objective bedside test which has established population norms [9-13] and has been extensively tested in a range of chronic general medical conditions [14]. It may

\section{() Biomed Central}


reflect, in part, the association of impaired muscle strength with malnutrition [15] and cardiopulmonary or metabolic diseases [4-7]. Hand grip strength can be assessed by instructing the patient to keep their shoulders adducted and neutrally rotated, the arm in a vertical position, the wrist in a neutral position and to squeeze the grip with maximal strength. The highest result in a seated or semi-seated position may be used $[16,17]$. Whether a robust relationship between preoperative handgrip strength and postoperative outcomes exists is unclear, since variable, and frequently retrospective, definitions of postoperative morbidity have been employed as outcome measures [18]. Therefore, we performed a systematic review of the literature to ascertain if preoperative assessment of handgrip strength is associated with (i) postoperative morbidity, (ii) length of hospital stay.

\section{Methods}

The systematic review was undertaken in accordance with the PRISMA [19] (Preferred Reporting Items for Systematic reviews and Meta-Analyses) and MOOSE
(Meta-analysis of Observational Studies in Epidemiology) [20] guidelines. Figure 1 summarizes the flow of information through the different phases of this systematic review. A checklist demonstrating adherence to the PRISMA guidelines is available online (Additional File 1).

Two of the authors (P.S. and M.A.H.) searched the electronic databases MEDLINE, EMBASE, and the Cochrane Central Register of Controlled Clinical Trials independently using the following population search terms: 'postoperative complications' OR 'perioperative complications' OR 'surgical complications' OR 'surgical outcome'. These search results were combined with 'handgrip dynamometry' OR 'hand grip dynamometry' OR 'hand grip strength' OR 'handgrip strength' OR 'maximal voluntary contraction' in the title or abstract text using the Boolean search operator 'AND'. (Maximal voluntary contraction is the term used most commonly in the literature to describe maximal force produced by a muscle as it contracts while contracting against the hand dynamometer). The references of retrieved articles were

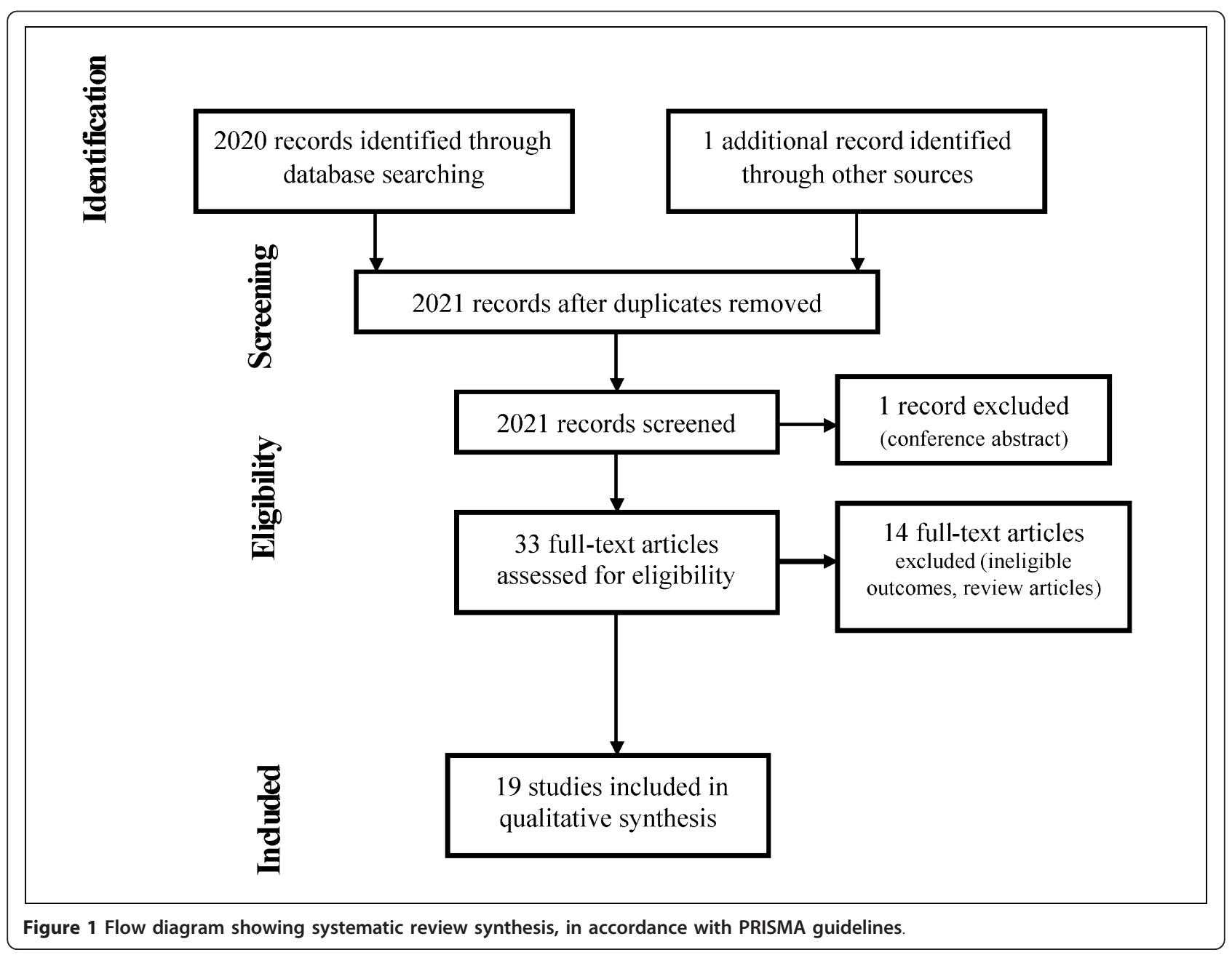


hand searched for any relevant articles not identified in the original search. The study selection criteria were limited to include only studies reported in the English language and those involving human adults undergoing surgery (including cardiac and transplant surgery). Each abstract was screened to identify studies that had assessed handgrip strength prior to surgery. Studies were excluded if postoperative outcomes focussed on upper limb neuromuscular functional outcomes alone.

The data were extracted on to a standardized data entry form by each reviewer. Differences between the reviewers were resolved by re-examination of the original manuscript until consensus was obtained. Data extracted for comparison included year of publication, primary author, total number of subjects, mean patient age, proportion of male subjects and co-morbidity (where reported). The method of quantifying or qualifying handgrip strength was recorded.

The specific outcomes sought in each article were: (i) mortality, (ii) postoperative morbidity, categorized according to the Post Operative Morbidity Survey, (iii) length of hospital stay [21]. Primary and/or secondary outcomes were recorded according to the a priori intention of each original article. Each outcome was evaluated qualitatively according to either qualitative and/or quantitative assessment of handgrip strength. Because there were a limited number of studies with homogenous design for each outcome, a meta-analysis could not be performed.

The procedural quality of each trial was assessed using several criteria, although no studies were excluded on the basis of these assessments. The quality of studies was scored according to the Newcastle-Ottawa Quality Assessment Scale [22] (Additional File 2), on a scale from 1 (poor) to 8 (excellent), which includes patient follow-up rates as a marker of study quality. Disagreements about the eligibility of a study or differences between the two sets of information extracted were resolved through discussion between all authors. After abstraction of information, a level of evidence was assigned to the outcomes of each study. Two authors (P.S. and M.A.H.) independently reviewed and scored each study using this method.

\section{Results}

Nineteen studies were identified that compared postoperative outcomes in relation to handgrip strength (Table 1), comprising 2194 patients [9-13,16,17,23-34]. A wide range of surgical sub-specialties was explored. Four studies were conducted in patients undergoing urgent surgery for hip fractures. One study explored the effect of pre-operative nutritional supplementation on grip strength [25]. Although supplementation improved post-operative grip strength compared to the control group, it was not related to patient outcome. Only two studies ensured that investigators who evaluated postoperative morbidity also remained blinded to the pre-operative grip strength values [10,17]. A wide range of exclusion criteria were reported between studies. One-third of studies reported the patient drop-out rate.

The majority of studies measured handgrip strength preoperatively (Table 2). Eleven studies did not comment on how long before surgery the handgrip strength was measured [9,10,17,24,25,28,30-33]. Guo et al did not comment on whether handgrip strength was measured pre or postsurgery [11]. Very few studies achieved a quality assessment score less than 6 , consistent with moderate quality (Table 3, Additional File 1).

\section{Definition of impaired handgrip strength}

Variable definitions for impaired handgrip strength have been used across studies (Table 4). Studies compared values of grip strength obtained from healthy controls, reference populations or patients who did not sustain postoperative morbidity with surgical patients. For example, 9 studies defined impaired handgrip strength as $<85 \%$ of a general, age-matched population - but these reference populations were not common between studies. Table 1 demonstrates that six studies measured handgrip strength exclusively from the non-dominant hand, compared to 3 studies that measured handgrip strength in the dominant hand. Seven studies did not report which hand was tested. $11 / 19$ studies did not report the timespan over which handgrip strength measurements preceded surgery. Variable time points were used between studies to assess postoperative handgrip strength. Detailed protocols for the performance of handgrip strength were absent in the majority of studies.

\section{Postoperative morbidity}

Table 5 summarizes the 15 studies that detailed the relationship between handgrip strength and various aspects of postoperative morbidity. Ten out of these 15 studies described a significant relationship between lower handgrip strength and postoperative morbidity $[9,10,12,13,25,28,30-33]$. No studies defined postoperative morbidity using validated morbidity tools. A range of morbidities were recorded prospectively: very few studies defined in detail how these morbidities were determined. Five studies used length of hospital stay as a surrogate for postoperative complications, but did not describe the associated morbidities.

\section{Length of Hospital Stay}

Tables 5 and 6 show the 12 studies which utilised length of stay as an outcome measure for postoperative morbidity. Five of these studies incorporated length of hospital stay into their definition of "complications" [9,28,30,32,33] and 7 studies separately explored the relationship between handgrip strength and length of hospital stay $[10,11,13,17$, 
Table 1 Basic demographics, defined primary/secondary outcomes and handgrip site used for patient studies.

\begin{tabular}{|c|c|c|c|c|c|c|c|c|c|}
\hline Author & Year & $\begin{array}{l}\text { Study } \\
\text { type }\end{array}$ & $\begin{array}{c}\text { Surgery } \\
\text { (urgency/type) }\end{array}$ & $\begin{array}{l}\text { Number of } \\
\text { patients } \\
\text { (n) }\end{array}$ & $\begin{array}{c}\text { Age } \\
\text { (mean } \pm \\
\text { SD or } \\
\text { mean } \\
\text { [range]) }\end{array}$ & $\begin{array}{l}\text { Gender } \\
(\% \text { male) }\end{array}$ & $\begin{array}{l}\text { Primary } \\
\text { Outcome }\end{array}$ & $\begin{array}{l}\text { Secondary } \\
\text { Outcome }\end{array}$ & $\begin{array}{c}\text { Handgrip: } \\
\text { dominant } \\
\text { vs. } \\
\text { non- } \\
\text { dominant? }\end{array}$ \\
\hline $\begin{array}{l}\text { Beloosesky } \\
\quad[16]\end{array}$ & 2010 & Cohort $^{*}$ & Urgent fractured neck of femur & 105 & $81 \pm 7$ & 31 & Functional outcome & Not stated & Dominant \\
\hline Wehern [23] & 2005 & Cohort & Urgent Hip fracture & 205 & $81 \pm 8$ & 0 & Functional outcome & Not stated & Right arm \\
\hline $\begin{array}{l}\text { Mahalakshmi } \\
{[10]}\end{array}$ & 2004 & $\begin{array}{l}\text { Case } \\
\text { control }\end{array}$ & Elective general & 100 & $42[13-70]$ & 62 & Complications & Not stated & $\begin{array}{l}\text { Non } \\
\text { dominant }\end{array}$ \\
\hline Cook [17] & 2001 & $\begin{array}{l}\text { Case } \\
\text { control }\end{array}$ & Elective CABG & 200 & Not stated & 73 & Complications & Not stated & Both hands \\
\hline $\begin{array}{l}\text { Figueiredo } \\
\text { [24] }\end{array}$ & 2000 & Cohort & Elective Liver transplant & 53 & $50 \pm 12$ & 59 & Complications & Not stated & Both hands \\
\hline Le Cornu [25] & 2000 & $\begin{array}{l}\text { Case } \\
\text { control }\end{array}$ & Elective liver transplant & 82 & $24-68$ & 73 & Complications & Not stated & Not stated \\
\hline Visser [26] & 2000 & Cohort & Urgent Hip fracture & 90 & $79 \pm 8$ & 0 & Mobility & Not stated & Not stated \\
\hline Guo [11] & 1996 & $\begin{array}{l}\text { Case } \\
\text { control }\end{array}$ & Elective oral and maxillofacial cancers & 127 & $54 \pm 15$ & 69 & Complications & Not stated & $\begin{array}{l}\text { Non } \\
\text { dominant }\end{array}$ \\
\hline Watters [27] & 1993 & Cohort & Elective general & 40 & $\begin{array}{c}<50 y \\
\text { group (36 } \pm \\
9) \\
>70 y \\
\text { group ( } 77 \pm \\
\text { 5) }\end{array}$ & 65 & $\begin{array}{l}\text { Relate Muscle strength to body } \\
\text { composition and nitrogen balance }\end{array}$ & Not stated & $\begin{array}{c}\text { Non } \\
\text { dominant }\end{array}$ \\
\hline $\begin{array}{c}\text { Schroeder } \\
\text { [34] }\end{array}$ & 1993 & Cohort & Elective general & 84 & $54 \pm 18$ & 44 & Post-op fatigue & Not stated & Dominant \\
\hline Griffith [28] & 1989 & Cohort & Elective general and vascular & 61 & $66^{\#[41-82]}$ & 75 & Complications & Not stated & Dominant \\
\hline $\begin{array}{l}\text { Kalfarentzos } \\
\quad[12]\end{array}$ & 1989 & $\begin{array}{l}\text { Case } \\
\text { control }\end{array}$ & Elective general & 95 & 70 [42-88] & 56 & Complication & Not stated & Not stated \\
\hline Brenner [29] & 1989 & Cohort & Elective general and vascular & 249 & Not stated & 66 & Complications & Not stated & Not stated \\
\hline Webb [30] & 1989 & $\begin{array}{l}\text { Case } \\
\text { control }\end{array}$ & Elective general & 90 & 58 [20-88] & 60 & Complications & Not stated & Not stated \\
\hline Shukla [31] & 1987 & $\begin{array}{l}\text { Case } \\
\text { control }\end{array}$ & Elective Major general & 110 & $20-70$ & 49 & Complications & Not stated & $\begin{array}{c}\text { non } \\
\text { dominant }\end{array}$ \\
\hline Hunt [13] & 1985 & $\begin{array}{l}\text { Case } \\
\text { control }\end{array}$ & $\begin{array}{l}\text { General, Orthopedic, Urology, Gynaecology, } \\
\text { Cardiovascular, Endocrine and Miscellaneous }\end{array}$ & 205 & $45 \pm 17$ & 46 & Complications & Not stated & Not stated \\
\hline Davies [32] & 1984 & Cohort & Urgent Fracture neck of femur & 76 & Not stated & Female & Complications & Not stated & Not stated \\
\hline Klidjian [33] & 1982 & $\begin{array}{l}\text { Case } \\
\text { control }\end{array}$ & Elective general & 120 & $60[24-86]$ & 55 & Complications & Not stated & $\begin{array}{l}\text { Non } \\
\text { dominant }\end{array}$ \\
\hline Klidjian [9] & 1980 & $\begin{array}{l}\text { Case } \\
\text { control }\end{array}$ & Elective general & 102 & $57[16-81]$ & 46 & Complications & $\begin{array}{l}\text { Factors impairing } \\
\text { handgrip strength }\end{array}$ & $\begin{array}{l}\text { Non } \\
\text { dominant }\end{array}$ \\
\hline
\end{tabular}

*Retrospective study; \# median value. 
Table 2 Timing of handgrip measurements in patient studies.

\begin{tabular}{ccc}
\hline Author & Year & Timing of measurement \\
\hline Beloosesky [16] & 2010 & 7-10 days and 1, 3,6 months post-op \\
Wehern [23] & 2005 & During hospitalisation and 2,6, 12 months post-op \\
Mahalakshmi [10] & 2004 & Pre-op- timing not specified \\
Cook [17] & 2001 & Pre-op- timing not specified \\
Figueiredo [24] & 2000 & Pre-op- timing not specified \\
Le Cornu [25] & 2000 & Pre-op- timing not specified \\
Visser [26] & 2000 & 2-10 days and 12 months following admission \\
Guo [11] & 1996 & Not specified whether pre or post-surgery \\
Watters [27] & 1993 & Pre-op on day of surgery \\
Schroeder [34] & 1993 & Pre-op- timing not specified \\
Griffith [28] & 1989 & 2-3 days pre-op \\
Kalfarentzos [12] & 1989 & 2 days pre-op \\
Brenner [29] & 1989 & Pre-op- timing not specified \\
Webb [30] & 1989 & Pre-op- timing not specified \\
Shukla [31] & 1987 & 12-72 hours pre-op \\
Hunt [13] & 1985 & Pre-op- timing not specified \\
Davies [32] & 1984 & Pre-op- timing not specified \\
Klidjian [33] & 1982 & Pre-op- timing not specified \\
Klidjian [9] & 1980 &
\end{tabular}

24,25,27]. Three of these 7 studies reported an association between lower handgrip strength and prolonged length of stay $[10,13,25]$. Mean or median values were compared rather than log-rank analysis.

\section{Mortality}

Table 7 summarizes the 5 studies that explored the relationship between handgrip strength and postoperative mortality. Variable time points for postoperative

Table 3 Newcastle -Ottawa Quality Assessment Scores (NOS score).

\begin{tabular}{|c|c|c|c|c|c|c|c|c|c|c|c|}
\hline \multirow[b]{2}{*}{ Study } & \multirow[b]{2}{*}{ Year } & \multirow[b]{2}{*}{ Study type } & \multirow[b]{2}{*}{ NOS score } & \multicolumn{4}{|c|}{ Selection } & \multirow{2}{*}{$\begin{array}{c}\text { Comparability } \\
1\end{array}$} & \multicolumn{2}{|c|}{ Outcome } & \multirow[b]{2}{*}{3} \\
\hline & & & & 1 & 2 & 3 & 4 & & 1 & 2 & \\
\hline Beloosesky [16] & 2010 & Cohort & 6 & $D$ & $a^{*}$ & $a^{*}$ & $a^{*}$ & & $b^{*}$ & $a^{*}$ & $a^{*}$ \\
\hline Wehren [23] & 2005 & Cohort & 6 & $a^{*}$ & $a^{*}$ & $b^{*}$ & $a^{*}$ & & $b^{*}$ & $a^{*}$ & $c<60 \%$ \\
\hline Figueiredo [24] & 2000 & Cohort & 6 & D & $a^{*}$ & $a^{*}$ & $a^{*}$ & & $b^{*}$ & $a^{*}$ & $a^{*}$ \\
\hline Visser [26] & 2000 & Cohort & 6 & D & $a^{*}$ & $a^{*}$ & $a^{*}$ & & $b^{*}$ & $a^{*}$ & $a^{*}$ \\
\hline Watters [27] & 1993 & Cohort & 7 & $a^{*}$ & $a^{*}$ & $b^{*}$ & $a^{*}$ & & $b^{*}$ & $a^{*}$ & $a^{*}$ \\
\hline Schroeder [34] & 1993 & Cohort & 6 & D & $a^{*}$ & $b^{*}$ & $a^{*}$ & & $b^{*}$ & $a^{*}$ & $a^{*}$ \\
\hline Griffith [28] & 1989 & Cohort & 7 & $a^{*}$ & $a^{*}$ & $b^{*}$ & $a^{*}$ & & $b^{*}$ & $a^{*}$ & $a^{*}$ \\
\hline Brenner [29] & 1989 & Cohort & 6 & D & $a^{*}$ & $b^{*}$ & $a^{*}$ & & $b^{*}$ & $a^{*}$ & $a^{*}$ \\
\hline Davies [32] & 1984 & Cohort & 7 & $a^{*}$ & $a^{*}$ & $b^{*}$ & $a^{*}$ & & $b^{*}$ & $a^{*}$ & $a^{*}$ \\
\hline Mahalakshmi [10] & 2004 & Case-control & 7 & $a^{*}$ & $a^{*}$ & $b^{*}$ & $a^{*}$ & & $b^{*}$ & $a^{*}$ & $a^{*}$ \\
\hline Cook [17] & 2001 & Case-control & 7 & $a^{*}$ & $a^{*}$ & $b^{*}$ & $a^{*}$ & & $b^{*}$ & $a^{*}$ & $a^{*}$ \\
\hline Le Cornu [25] & 2000 & Case-control & 7 & $a^{*}$ & $a^{*}$ & $b^{*}$ & $a^{*}$ & $a^{*}$ & c & $a^{*}$ & $a^{*}$ \\
\hline Guo [11] & 1996 & Case-control & 6 & $a^{*}$ & $a^{*}$ & $b^{*}$ & $a^{*}$ & & C & $a^{*}$ & $a^{*}$ \\
\hline Kalfarentzos [12] & 1989 & Case-control & 6 & $a^{*}$ & $a^{*}$ & $b^{*}$ & $a^{*}$ & & C & $a^{*}$ & $a^{*}$ \\
\hline Webb [30] & 1989 & Case-control & 4 & C & $b$ & $b^{*}$ & $a^{*}$ & & c & $a^{*}$ & $a^{*}$ \\
\hline Shukla [31] & 1987 & Case-control & 6 & $a^{*}$ & $a^{*}$ & $b^{*}$ & $a^{*}$ & & C & $a^{*}$ & $a^{*}$ \\
\hline Hunt [13] & 1985 & Case-control & 4 & C & $b$ & $b^{*}$ & $a^{*}$ & & c & $a^{*}$ & $a^{*}$ \\
\hline Klidjian [33] & 1982 & Case-control & 4 & C & $b$ & $b^{*}$ & $a^{*}$ & & C & $a^{*}$ & $a^{*}$ \\
\hline Klidjian [9] & 1980 & Case-control & 4 & C & $b$ & $b^{*}$ & $a^{*}$ & & C & $a^{*}$ & $a^{*}$ \\
\hline
\end{tabular}

Letters represent answer for corresponding numbered question in each section. A study can be awarded a maximum of one star for each numbered item within the Selection and Outcome categories. A maximum of two stars can be given for Comparability. See Additional File 1 for full details of assessment criteria. 
Table 4 Definitions used for impaired handgrip Strength.

\begin{tabular}{|c|c|c|c|}
\hline Author & $\begin{array}{l}\text { A priori definition of Impaired handgrip } \\
\text { strength? }\end{array}$ & $\begin{array}{l}\text { Definition of impaired handgrip } \\
\text { strength }\end{array}$ & Post-hoc Definition/comparison \\
\hline $\begin{array}{l}\text { Beloosesky } \\
{[16]}\end{array}$ & $\mathrm{NO}$ & & $\begin{array}{l}\text { Functional Independence Measure } 6 \text { months } \\
\text { postoperatively }\end{array}$ \\
\hline Wehren [23] & NO & & Activities of Daily Living \\
\hline $\begin{array}{l}\text { Mahalakshmi } \\
{[10]}\end{array}$ & YES & $<85 \%$ control values & \\
\hline Cook [17] & NO & & According to low or high risk status \\
\hline Figueiredo [24] & NO & & Critical Care length of stay \\
\hline Le Cornu [25] & NO & & $<85 \%$ and $>85 \%$ \\
\hline Visser [26] & NO & & Loss in grip strength post-operatively \\
\hline Guo [11] & YES & $<85 \%$ control values & \\
\hline Watters [27] & NO & & Loss in grip strength post-operatively \\
\hline Schroeder [34] & NO & & Post-operative fatigue \\
\hline Griffith [28] & NO & & Loss in grip strength post-operatively \\
\hline $\begin{array}{l}\text { Kalfarentzos } \\
{[12]}\end{array}$ & YES & $<85 \%$ control values & \\
\hline Brenner [29] & NO & & \\
\hline Webb [30] & YES & $<85 \%$ population norm & \\
\hline Shukla [31] & NO & & $<85 \%$ and $>85 \%$ \\
\hline Hunt [13] & YES & $<85 \%$ healthy controls & \\
\hline Davies [32] & NO & & $<15 \mathrm{~kg}$ \\
\hline Klidjian [33] & YES & $\begin{array}{l}<85 \% \text { controls } \\
{[1980 \text { study] }}\end{array}$ & \\
\hline Klidjian [9] & NO & & $<$ and $>85 \%$ \\
\hline
\end{tabular}

associated death were defined across studies, ranging from 30 days to 6 month mortality following surgery. One study did not define the time period of follow-up for patients to determine mortality. Two studies reported an association between lower handgrip strength and increased mortality [12,17].

\section{Discussion}

Contrary to large population studies, our systematic review of the relationship between preoperative handgrip strength and postoperative outcome did not find compelling data to support the hypothesis that the results of studies in the general population translate to

Table 5 Type of Postoperative morbidity included in follow-up of patients.

\begin{tabular}{|c|c|c|c|c|c|c|c|c|c|}
\hline & Pulmonary & Infectious & Renal & Gastrointestinal & Cardiovasular & Neurological & Wound & Haematological Pain & LOS \\
\hline Mahalakshmi [10] & Y & Y & Y & Y & Y & Y & Y & & Sep \\
\hline Cook [17] & $\mathrm{Y}$ & $\mathrm{Y}$ & $\mathrm{Y}$ & & $Y$ & & & & Sep \\
\hline Figueiredo[24] & & Y & & & & & & $\mathrm{Y}$ & Sep \\
\hline Le Cornu[25] & $\mathrm{Y}$ & $\mathrm{Y}$ & & & & & & & Sep \\
\hline Guo[11] & & Y & & & & & Y & & Sep \\
\hline Watters[27] & & & & & & & & & Sep \\
\hline \multicolumn{10}{|l|}{ Schroeder[34] } \\
\hline Griffith[28] & Y & Y & & & Y & & & & $Y \neq$ \\
\hline Brenner[29] & Y & Y & & & & & Y & & \\
\hline$\overline{\text { Webb[30] }}$ & & & & & & & & & $Y^{*}$ \\
\hline Shukla[31] & Y & & & & Y & & Y & & \\
\hline Hunt[13] & Y & Y & Y & Y & & & Y & & Sep \\
\hline Davies[32] & & & & & & & & & $\mathrm{Y}+$ \\
\hline Klidjian[33] & Y & Y & & & & & & & $Y^{*}$ \\
\hline Klidjian[9] & & & & & & & & & $Y^{*}$ \\
\hline
\end{tabular}


Table 6 Studies describing relationship between Grip Strength (GS) and Hospital Length of Stay (LOS).

\begin{tabular}{|c|c|c|c|c|c|c|}
\hline AUTHOR & $\begin{array}{l}\text { GS of LOS "Controls" } \\
\text { (kg or \%) }\end{array}$ & $\begin{array}{c}\text { Control LOS } \\
\text { (mean days } \pm \text { SD) }\end{array}$ & $\begin{array}{c}\text { GS of LOS "comparators" } \\
\text { (kg or \%) }\end{array}$ & $\begin{array}{c}\text { Comparator LOS (mean } \\
\text { days } \pm \text { SD) }\end{array}$ & $\begin{array}{l}\text { Log-rank } \\
\text { test? }\end{array}$ & $\begin{array}{l}\text { LOS and Handgrip strength } \\
\text { associated? }\end{array}$ \\
\hline $\begin{array}{l}\text { Mahalakshmi } \\
{[10]}\end{array}$ & $<85 \%$ & $12.8 \pm 6.6$ & $>85 \%$ & $9.3 \pm 3.4$ & $\mathrm{NO}$ & YES \\
\hline Cook [17] & $\begin{aligned} \text { Male } & <32 \mathrm{~kg} \\
\text { Female } & <20.5 \mathrm{~kg}\end{aligned}$ & $8.1 \pm 10$ & $\begin{aligned} \text { Male } & >32 \mathrm{~kg} \\
\text { Female } & >20.5 \mathrm{~kg}\end{aligned}$ & $6.8 \pm 7.5$ & NO & NO \\
\hline $\begin{array}{c}\text { Figueiredo } \\
\text { [24] }\end{array}$ & ICU stay only* & Not presented & Not presented & Not presented & $\mathrm{NO}$ & $\mathrm{NO}$ \\
\hline Le Cornu [25] & Not presented & Not presented & Not presented & Not presented & YESt & Positive correlation \\
\hline Guo [11] & $<85 \%$ & $42 \pm 20$ & $>85 \%$ & $32 \pm 10$ & NO & \\
\hline Watters [27] & Not presented & Not presented & Not presented & Not presented & NO & NO \\
\hline Hunt [13] & $<85 \%$ & $11.4 \pm 12$ & $>85 \%$ & $6.8 \pm 3.8$ & NO & YES \\
\hline Griffith [28] & $\begin{array}{c}\text { Mean: } \\
\text { Male } 25.4 \pm 9.1 \mathrm{~kg} \\
\text { Female } 14.4 \pm 4.3 \mathrm{~kg}\end{array}$ & $\begin{array}{l}\text { 7/61 had "Complications" (definition included } \\
\text { LOS > } 14 \text { days) }\end{array}$ & $\begin{array}{c}\text { Mean: } \\
\text { Male } 30.2 \pm 8.4 \mathrm{~kg} \\
\text { Female } 14.9 \pm 5.7 \mathrm{~kg}\end{array}$ & 48/61 had LOS $<14$ days & $\mathrm{NO}$ & Not reported \\
\hline Webb [30] & $<85 \%$ & $\begin{array}{l}\text { 20/51 had "complications" (definition included } \\
\text { LOS > } 14 \text { days) }\end{array}$ & $>85 \%$ & $7 / 39$ had LOS $<14$ days & $\mathrm{NO}$ & Not reported \\
\hline Davies [32] & $<15 \mathrm{~kg}$ & $\begin{array}{l}\text { 27/37 had "Complications" (definition included } \\
\text { LOS > } 16 \text { days) }\end{array}$ & $>15 \mathrm{~kg}$ & $3 / 14$ had LOS $<16$ days & $\mathrm{NO}$ & Not reported \\
\hline Klidjian [33] & $<85 \%$ & $\begin{array}{l}\text { 43/72 had "complications" (definition included } \\
\text { LOS }>14 \text { days) }\end{array}$ & $>85 \%$ & $5 / 48$ had LOS $<14$ days & $\mathrm{NO}$ & Not reported \\
\hline Klidjian [9] & $<85 \%$ & $\begin{array}{c}\text { 20/44 had "complications" (definition included } \\
\text { LOS }>14 \text { days) }\end{array}$ & $>85 \%$ & $3 / 58$ had LOS $<14$ days & $\mathrm{NO}$ & Not reported \\
\hline
\end{tabular}

*Increased ITU stay was associated with lower handgrip strength (right (27 \pm 6 and $36 \pm 12 \mathrm{~kg} \mathrm{p}<0.01$ ) and left $(27 \pm 7$ and $35 \pm 12 \mathrm{~kg} p=0.01)$

tThere was a correlation between grip strength and day of discharge post-transplant $(r=-0.41, P=0.01)$. There was no association between grip strength and length of time spent on ventilatory support posttransplant $(r=-0.250)$ or length of time spent on the intensive care unit post-transplant $(r=-0.112)$

* Survival plot from time of listing to transplant or death (not for grip strength). 
Table 7 Studies describing relationship between handgrip strength and postoperative mortality.

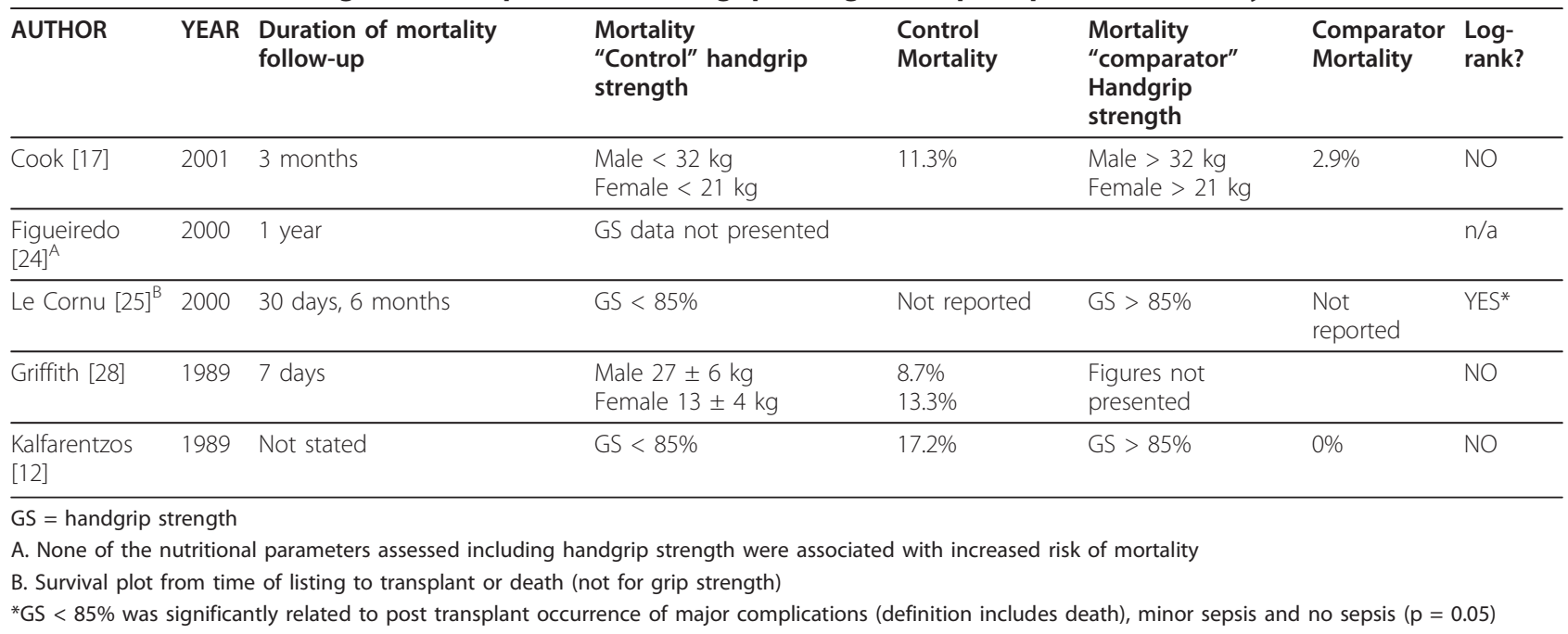

perioperative medicine. The majority of studies were considered to be of reasonable quality. Despite these quality scores, many studies contained important potential confounding factors which varied markedly between studies. A range of different instruments have been employed to measure grip strength, with other corroborative assessments of strength being frequently absent. Due to the substantial variation in the way in which each specified outcome had been defined between studies, plus the lack of analyses testing any one particular association, it was not possible to perform meta-analyses of results or formally test the heterogeneity (consistency) between studies. This marked heterogeneity between studies limits any definitive conclusions for the perioperative environment and renders this preoperative assessment largely unexplored. Nevertheless, several of these studies - albeit with the limitations as discussed above - suggest the role for preoperative handgrip strength assessment should be explored further.

Large epidemiological studies have shown that perioperative morbidity is associated with dramatic differences in post-discharge life expectancy across different operations and health systems [2]. The cost and expertise required by certain preoperative tests, such as cardiopulmonary exercise testing, plus other limiting factors (e.g. dysmobility, acuity of surgery) necessitates an alternative approach to be developed for the objective assessment of perioperative risk in the substantial minority of patients who may sustain morbidity that impacts on their longer-term survival. The development of an inexpensive, mass screening preoperative assessment tool with high sensitivity and specificity to detect postoperative morbidity is clearly attractive. Handgrip strength is an easy, non-invasive, cheap, real-time and established independent "bedside" predictor of long-term all-cause mortality in more than 44,000 patients studied in the general population [14].

There are also compelling basic biological reasons for establishing the role of handgrip strength in preoperative assessment. Cardiopulmonary reserve is a longestablished predictor of cardiovascular and all-cause mortality, in both asymptomatic individuals and patients with cardiovascular disease [35]. Cardiac insufficiency has emerged as the commonest preoperative morbidity associated with increased morbidity and mortality [36,37]. An important component of cardiac failure is dysfunctional skeletal muscle metabolism [38] and impaired strength - as reflected by handgrip strength [39]. Skeletal muscle exerts important effects on the patterns of substrate use during periods of increased cardiopulmonary performance $[40,41]$. Major alterations in skeletal muscle histology and biochemistry occur in patients with long-term heart failure [42,43]. These skeletal muscle adaptations may underlie the early onset of anaerobic metabolism, increased lactate production and fatigue in heart failure. Handgrip strength improves following specific interventions that increase cardiopulmonary reserve $[44,45]$. Muscle (handgrip) strength is also impaired in metabolic disease [46], which may in part explain its association with both poorer perioperative outcomes and all-cause mortality.

One limitation of this systematic review is that no original study data were retrieved, although given the heterogeneity of both study design and the surgical populations in question this would have been unlikely to alter the main conclusions. Because only published reports were examined (obtained from searches performed only on MEDLINE, EMBASE and Cochrane databases), a formal assessment of publication bias was not undertaken. It remains possible that not all relevant studies may have 
been identified since unpublished studies were not sought. There is very little perioperative demographic data provided in these studies, including cardiovascular risk and the identification of higher risk patients. Standards of postoperative care were not reported or apparently standardized. Since no interventions were conducted based on preoperative handgrip strength assessment, the studies only provide associative conclusions.

This systematic review has generated two significant clinical implications. Firstly, given the compelling general population data that predicts longevity, there is clearly a need for the further prospective assessment of whether preoperative handgrip strength can help stratify risk of adverse postoperative outcomes. Second, these studies demonstrate that handgrip strength is a feasible, pragmatic, real-time bedside tool that may enhance preoperative risk stratification.

\section{Conclusions}

Impaired preoperative handgrip strength may be associated with increased postoperative morbidity, mortality and prolonged hospital stay following surgery. Given the robust predictive power of this inexpensive, objective bedside test beyond the perioperative population, further studies of its' role in predicting postoperative outcomes appear to be warranted provided prospective, objectively defined measures of morbidity are employed.

\section{Additional material}

Additional file 1: Checklist of items demonstrating adherence to PRIMSA systematic review guidelines.

Additional file 2: Newcastle Ottowa Scale.

\begin{abstract}
Acknowledgments and funding
Centre for Anaesthesia, Critical Care and Pain Management, University College London, UK [PS, MAH]; Academy of Medical Sciences/Health Foundation Clinician Scientist award [GLA]. This work was undertaken at UCLH/UCL who received a proportion of funding from the Department of Health's NIHR Biomedical Research Centres funding scheme.

Author details

'Department of Anesthesia, Stanford University Medical Center, Stanford, California 94305, USA. ${ }^{2}$ Anaesthesia and Critical Care, St Georges Hospital NHS Trust, London, UK. ${ }^{3}$ Centre for Anaesthesia, Critical Care and Pain Management, University College London, London, UK. ${ }^{4}$ Department of Medicine, University College London, London, UK.
\end{abstract}

\section{Authors' contributions}

All authors contributed to Study design, Conduct of study, Data analysis and Manuscript preparation.

\section{Competing interests}

The authors declare that they have no competing interests.

Received: 23 May 2011 Accepted: 17 January 2012

Published: 17 January 2012
References

1. Ackland GL, Edwards M: Defining higher-risk surgery. Curr Opin Crit Care 2010, 16(4):339-346.

2. Khuri SF, Henderson WG, DePalma RG, Mosca C, Healey NA, Kumbhani DJ: Determinants of long-term survival after major surgery and the adverse effect of postoperative complications. AnnSurg 2005, 242(3):326-341.

3. Pearse R, Harrison D, James P, Watson D, Hinds C, Rhodes A, Grounds RM, Bennett ED: Identification and characterisation of the high-risk surgical population in the United Kingdom. Critical Care 2006, 10(3):R81-R81.

4. Marzetti $E$, Leeuwenburgh C: Skeletal muscle apoptosis, sarcopenia and frailty at old age. ExpGerontol 2006, 41(12):1234-1238.

5. Kuh D, Bassey EJ, Butterworth S, Hardy R, Wadsworth ME: Grip strength, postural control, and functional leg power in a representative cohort of British men and women: associations with physical activity, health status, and socioeconomic conditions. J Gerontol A Biol Sci Med Sci 2005, 60(2):224-231.

6. Klein BE, Klein R, Knudtson MD, Lee KE: Frailty, morbidity and survival. ArchGeronto/Geriatr 2005, 41(2):141-149.

7. Morris CK, Ueshima K, Kawaguchi T, Hideg A, Froelicher VF: The prognostic value of exercise capacity: a review of the literature. Am Heart J 1991, 122(5):1423-1431.

8. Smith TB, Stonell C, Purkayastha S, Paraskevas P: Cardiopulmonary exercise testing as a risk assessment method in non cardio-pulmonary surgery: a systematic review. Anaesthesia 2009, 64(8):883-893.

9. Klidjian AM, Foster KJ, Kammerling RM, Cooper A, Karran SJ: Relation of anthropometric and dynamometric variables to serious postoperative complications. BrMed J 1980, 281(6245):899-901.

10. Mahalakshmi VN, Ananthakrishnan N, Kate V, Sahai A, Trakroo M: Handgrip strength and endurance as a predictor of postoperative morbidity in surgical patients: can it serve as a simple bedside test? IntSurg 2004, 89(2):115-121

11. Guo CB, Zhang W, Ma DQ, Zhang KH, Huang JQ: Hand grip strength: an indicator of nutritional state and the mix of postoperative complications in patients with oral and maxillofacial cancers. $\mathrm{Br} J$ Oral Maxillofac Surg 1996, 34(4):325-327.

12. Kalfarentzos F, Spiliotis J, Velimezis G, Dougenis D, Androulakis J: Comparison of forearm muscle dynamometry with nutritional prognostic index, as a preoperative indicator in cancer patients. JPEN J Parenter Enteral Nutr 1989, 13(1):34-36.

13. Hunt DR, Rowlands BJ, Johnston D: Hand grip strength-a simple prognostic indicator in surgical patients. JPEN J Parenter Enteral Nutr 1985, 9(6):701-704.

14. Cooper R, Kuh D, Hardy R: Objectively measured physical capability levels and mortality: systematic review and meta-analysis. BMJ: British Medical Journal 2010, 341:c4467.

15. Wang AY, Sea MM, Ho ZS, Lui SF, Li PK, Woo J: Evaluation of handgrip strength as a nutritional marker and prognostic indicator in peritoneal dialysis patients. Am J Clin Nutr 2005, 81(1):79-86.

16. Beloosesky $Y$, Weiss A, Manasian M, Salai M: Handgrip strength of the elderly after hip fracture repair correlates with functional outcome. Disabil Rehabil 2010, 32(5):367-373.

17. Cook JW, Pierson LM, Herbert WG, Norton HJ, Fedor JM, Kiebzak GM, Ramp WK, Robicsek F: The influence of patient strength, aerobic capacity and body composition upon outcomes after coronary artery bypass grafting. Thorac Cardiovasc Surg 2001, 49(2):89-93.

18. Bohannon RW: Hand-grip dynamometry predicts future outcomes in aging adults. J Geriatr Phys Ther 2008, 31(1):3-10.

19. Moher D, Liberati A, Tetzlaff J, Altman DG: Preferred reporting items for systematic reviews and meta-analyses: the PRISMA statement. BMJ 2009, 339:b2535.

20. Stroup DF, Berlin JA, Morton SC, Olkin I, Williamson GD, Rennie D, Moher D, Becker BJ, Sipe TA, Thacker SB: Meta-analysis of observational studies in epidemiology: a proposal for reporting. Meta-analysis Of Observational Studies in Epidemiology (MOOSE) group. JAMA 2000, 283(15):2008-2012.

21. Bennett-Guerrero E, Welsby I, Dunn TJ, Young LR, Wahl TA, Diers TL, Phillips-Bute BG, Newman MF, Mythen MG: The use of a postoperative morbidity survey to evaluate patients with prolonged hospitalization after routine, moderate-risk, elective surgery. AnesthAnalg 1999, 89(2):514-519.

22. The Newcastle-Ottawa Quality Assessment Scale. 2009 [http:// wwwohrica/programs/clinical_epidemiology/nosgenpdf]. 
23. Wehren LE, Hawkes WG, Hebel JR, Orwig DL, Magaziner J: Bone mineral density, soft tissue body composition, strength, and functioning after hip fracture. J Gerontol A Biol Sci Med Sci 2005, 60(1):80-84.

24. Figueiredo F, Dickson ER, Pasha T, Kasparova P, Therneau T, Malinchoc M, DiCecco S, Francisco-Ziller N, Charlton M: Impact of nutritional status on outcomes after liver transplantation. Transplantation 2000, 70(9):1347-1352.

25. Le Cornu KA, McKiernan FJ, Kapadia SA, Neuberger JM: A prospective randomized study of preoperative nutritional supplementation in patients awaiting elective orthotopic liver transplantation. Transplantation 2000, 69(7):1364-1369.

26. Visser M, Harris TB, Fox KM, Hawkes W, Hebel JR, Yahiro JY, Michael R, Zimmerman SI, Magaziner J: Change in muscle mass and muscle strength after a hip fracture: relationship to mobility recovery. J Gerontol A Biol Sci Med Sci 2000, 55(8):M434-440.

27. Watters JM, Clancey SM, Moulton SB, Briere KM, Zhu JM: Impaired recovery of strength in older patients after major abdominal surgery. Ann Surg 1993, 218(3):380-390, discussion 390-383.

28. Griffith CD, Whyman M, Bassey EJ, Hopkinson BR, Makin GS: Delayed recovery of hand grip strength predicts postoperative morbidity following major vascular surgery. BrJ Surg 1989, 76(7):704-705.

29. Brenner U, Wolters U, Said S, Muller JM: Anthropometric parameters for preoperative screening to predict high-risk patients. Acta Chir Belg 1989 89(1):19-24.

30. Webb AR, Newman LA, Taylor M, Keogh JB: Hand grip dynamometry as a predictor of postoperative complications reappraisal using age standardized grip strengths. JPEN J Parenter Enteral Nutr 1989, 13(1):30-33.

31. Shukla HS, Ahmad MI, Shukla PK, Panda BK: Preoperative nutritional assessment by hand grip dynamometry. Indian J Med Res 1987, 86:321-328.

32. Davies $C W$, Jones DM, Shearer JR: Hand grip-a simple test for morbidity after fracture of the neck of femur. J R SocMed 1984, 77(10):833-836.

33. Klidjian AM, Archer TJ, Foster KJ, Karran SJ: Detection of dangerous malnutrition. JPEN J Parenter Enteral Nutr 1982, 6(2):119-121.

34. Schroeder D, Hill GL: Predicting postoperative fatigue: importance of preoperative factors. World J Surg 1993, 17(2):226-231.

35. Balady GJ, Arena R, Sietsema K, Myers J, Coke L, Fletcher GF, Forman D, Franklin B, Guazzi M, Gulati M, et al: Clinician's Guide to cardiopulmonary exercise testing in adults: a scientific statement from the American Heart Association. Circulation 2010, 122(2):191-225.

36. Hammill BG, Curtis LH, nett-Guerrero E, O'Connor CM, Jollis JG, Schulman KA, Hernandez AF: Impact of heart failure on patients undergoing major noncardiac surgery. Anesthesiology 2008, 108(4):559-567.

37. Hernandez AF, Whellan DJ, Stroud S, Sun JL, O'Connor CM, Jollis JG: Outcomes in heart failure patients after major noncardiac surgery. JAmCollCardiol 2004, 44(7):1446-1453.

38. Middlekauff HR: Making the case for skeletal myopathy as the major limitation of exercise capacity in heart failure. Circ Heart Fail 2010, 3(4):537-546.

39. Izawa KP, Watanabe S, Osada N, Kasahara Y, Yokoyama H, Hiraki K, Morio Y, Yoshioka S, Oka K, Omiya K: Handgrip strength as a predictor of prognosis in Japanese patients with congestive heart failure. Eur $J$ CardiovascPrevRehabil 2009, 16(1):21-27.

40. McGee SL, Hargreaves M: AMPK-mediated regulation of transcription in skeletal muscle. Clin Sci (Lond) 2010, 118(8):507-518.

41. Lessard SJ, Rivas DA, Chen ZP, van Denderen BJ, Watt MJ, Koch LG, Britton SL, Kemp BE, Hawley JA: Impaired skeletal muscle beta-adrenergic activation and lipolysis are associated with whole-body insulin resistance in rats bred for low intrinsic exercise capacity. Endocrinology 2009, 150(11):4883-4891.

42. Sullivan MJ, Green HJ, Cobb FR: Altered skeletal muscle metabolic response to exercise in chronic heart failure. Relation to skeletal muscle aerobic enzyme activity. Circulation 1991, 84(4):1597-1607.

43. Sullivan MJ, Green HJ, Cobb FR: Skeletal muscle biochemistry and histology in ambulatory patients with long-term heart failure. Circulation 1990, 81(2):518-527.

44. Nagaya N, Moriya J, Yasumura Y, Uematsu M, Ono F, Shimizu W, Ueno K, Kitakaze M, Miyatake K, Kangawa K: Effects of ghrelin administration on left ventricular function, exercise capacity, and muscle wasting in patients with chronic heart failure. Circulation 2004, 110(24):3674-3679.
45. O'Donnell DE, McGuire M, Samis L, Webb KA: General exercise training improves ventilatory and peripheral muscle strength and endurance in chronic airflow limitation. Am J Respir Crit Care Med 1998, 157(5 Pt 1):1489-1497.

46. Al SS, Markides KS, Ray L, Ostir GV, Goodwin JS: Handgrip strength and mortality in older Mexican Americans. J Am GeriatrSoc 2002, 50(7):1250-1256

\section{Pre-publication history}

The pre-publication history for this paper can be accessed here: http://www.biomedcentral.com/1471-2253/12/1/prepub

doi:10.1186/1471-2253-12-1

Cite this article as: Sultan et al.: Preoperative muscle weakness as defined by handgrip strength and postoperative outcomes:

a systematic review. BMC Anesthesiology 2012 12:1.

\section{Submit your next manuscript to BioMed Central and take full advantage of:}

- Convenient online submission

- Thorough peer review

- No space constraints or color figure charges

- Immediate publication on acceptance

- Inclusion in PubMed, CAS, Scopus and Google Scholar

- Research which is freely available for redistribution

Submit your manuscript at www.biomedcentral.com/submit
C) Biomed Central 